\title{
Crystal structure of TAPBPR/MHC-I complex reveals insights to the mechanism of peptide editing
}

\author{
Jiansheng Jiang ${ }^{1^{\star}}$, Kannan Natarajan ${ }^{1^{\star}}$, Lisa F. Boyd ${ }^{1}$, Giora I. Morozov ${ }^{2}$, \\ Michael G. Mage ${ }^{1}$, David H. Margulies ${ }^{1}$
}

${ }^{1}$ Molecular Biology Section, Laboratory of Immunology, National Institute of Allergy and Infectious Diseases, National Institutes of Health, Bethesda, MD. ${ }^{2}$ Faculty of Agriculture, Hebrew University, Rehovot, Israel

*These authors contributed equally

The cell surface expression of major histocompatibility complex class I (MHC-I) molecules presenting peptides is a critical requirement for $\mathrm{T}$ cell-mediated immunity. The mechanism of peptide selection and exchange, mediated primarily by tapasin in the peptide loading complex (PLC) remains elusive. A recently discovered tapasin-related protein, TAPBPR (TAP binding protein, related) was shown to function independently of the PLC by directly binding certain MHC-I alleles and facilitating peptide exchange. Small-angle X-ray scattering (SAXS) has shown that TAPBPR and tapasin share similar molecular shapes. To elucidate mechanistic details, we have examined the binding interaction between purified recombinant TAPBPR and MHC-I molecules loaded with short peptides that leave a portion of the peptide-binding groove unoccupied. An extensive crystallization screen with purified TAPBPR/MHC-I complexes yielded crystals that diffracted to 3.5 Angstrom resolution. The crystals were merohedrally twinned in the $\mathrm{P}_{1}$ space group. The structure of the

TAPBPR/MHC-I complex was solved by molecular replacement with partial domain models. Implementation of low resolution refinement protocols (DEN/CNS or PHENIX) facilitated the refinement and yielded a model with current R/Rfree of $0.25 / 0.28$. The structure of a TAPBPR/MHC-I complex provides insights into mechanisms of peptide loading that are also applicable to understanding tapasin function.

This research was supported by the intramural research program of the NIAID, NIH. 